\section{\$22. Magnetic Field Gradient and Curvature-Driven Drift Modes in Toroidal Plasmas}

Wang, A.K. (SWIP, China),

Sanuki, H., Dong, J.Q. (SWIP, China), Zonca, F.(ENEA C.R. Frascati), Itoh, K.

The observed transport coefficients for energy and particles generally exceed neoclassical prediction by more than two orders of magnitude. Understanding and controlling such anomalous transport is one of the major challenges for magnetic confinement fusion research. It is widely believed that such anomaly can be attributed to a variety of small scale turbulence driven by gradients of plasma parameters such as density, temperature, magnetic field and so on. Among them, the ion and electron temperature gradient instabilities have been extensively studied over the past years. In the basic slab geometry, these instabilities are essentially sound waves coupled to pressure fluctuations. In toroidal geometry, the instabilities are still dominated by temperature gradients but enhanced by unfavorable magnetic curvature. In addition to the ion and electron temperature gradients, it has kept an active research area to explore the instabilities dominated by other driving sources. In this work, we present a new kind of instability in toroidal plasmas: magnetic field gradient and curvature (MFGC)-driven drift modes. The MFGC drift modes have a basic feature. That is, they possess the finite growth rates, driven completely by magnetic field gradient and curvature, even if the plasma pressure gradient is zero. Hence, they are a kind of universal instability in toroidal magnetically confined plasmas.

To illustrate the existence of new kind of instability, we consider a quasi-neutrality tokamak plasma of circular cross-section with $\varepsilon=r / R \ll 1$ within the framework of two fluids in the sheared coordinate system. Typical results are plotted in Fig.1.

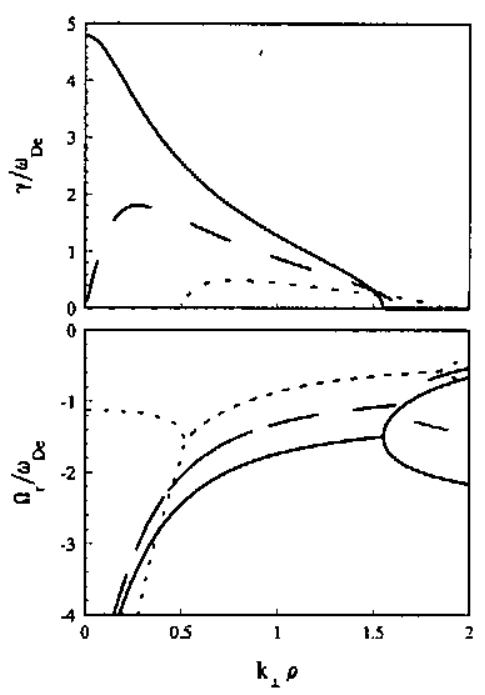

Fig.1 growth rate and real frequency versus $k_{\perp} \rho$ For $T_{i} / T_{e}=1.5$ (solid line), 1 (dashed) and 0.5 (dotted line). 